\title{
Understanding and characterizing the drug sorption to PVC and PE materials
}

\author{
Meriem Sahnoune, ${ }^{\dagger}$ Nicolas Tokhadzé, ${ }^{\dagger}$ Julien Devémy, ${ }^{\dagger}$ Alain Dequidt, ${ }^{\dagger}$ Florent \\ Goujon, ${ }^{\dagger}$ Philip Chennell, ${ }^{\ddagger}$ Valérie Sautou, ${ }^{\ddagger}$ and Patrice Malfreyt ${ }^{*, \dagger}$ \\ †Université Clermont Auvergne, CNRS, SIGMA Clermont, Institut de Chimie de \\ Clermont-Ferrand, F-63000 Clermont-Ferrand, France \\ $\ddagger$ Université Clermont Auvergne, CHU Clermont-Ferrand, CNRS, SIGMA Clermont, \\ Institut de Chimie de Clermont-Ferrand, F-63000 Clermont-Ferrand, France \\ E-mail: Patrice.Malfreyt@uca.fr
}

\section{Abstract}

Characterizing the sorption of drugs onto PVC and PE materials in terms of thermodynamic adsorption properties and atomistic details (local arrangements, orientation, diffusion) is fundamental for the development of alternatives materials that would limit drug sorption phenomena and plasticizer release. Here, a combination of experiments and sophisticated calculations of potential of mean forces are carried out to investigate the sorption of paracetamol and diazepam to PE and PVC surfaces. The simulated Gibbs free energies of adsorption are in line with the experimental interpretations. The polymer-drug-water interface is then characterized at the molecular scale by an in-depth investigation of local properties such as density, orientation and diffusion.

\section{Keywords}

sorption, thermodynamic properties, potential of mean force, molecular description, plastic materials, drug loss, drug-material interactions

\section{Introduction}

Plastic materials such as polyethylene (PE) and polyvinylchloride (PVC) are widely used in var- ious medical devices (MD) such as infusion lines for injectable drugs, artificial nutrition tubings, intubation, blood and plasma transfusion sets, catheters and syringes. ${ }^{1} \mathrm{PVC}$ is the most common material used for the manufacturing of infusion tubings because if its good mechanical properties, low manufacturing cost, clarity, transparency, recyclability and flexibility obtained with the addition of plasticizers. However, it presents some drawbacks, in particular because of it can release potentially toxic plasticizers (eg phthalates, known as endocrine disruptors) and can cause the sorption of infused medications, thus leading to drug loss and patient under-dosing. PE, a plasticizer-free material, has an overall better compatibility with infused drugs than PVC. However, it is less used than PVC to manufacture infusion tubings because of its rigidity and its opacity, making it difficult to clamp the tubings, generating kinks and not limiting visibility of the infused drug.

Sorption (including the adsorption of the drug to the polymer surface, absorption into the polymer matrix and permeation) can affect the efficacy, the loss of an important component, such as an active pharmaceutical ingredient $(\text { API })^{2-9}$ or an excipient. ${ }^{10}$ This can lead to ineffective or variable drug responses after the administration of injectable drugs, and makes it difficult to control the concentration of the delivered drug. A loss of isosorbide dinitrate, 
tacrolimus, diazepam, amiodarone, insulin has been observed during infusions via PVC tubings. ${ }^{2-9}$ These losses of API by sorption can require an adjustment of the dose administered, based on the expected biological effect of the drug. Sometimes, rinsing the tubing by the drug before its administration is a possible way to limit these interactions. This is the case with insulin. ${ }^{8-10}$ However, for hydrophobic APIs, like diazepam, a simple rinse is not enough. Drugpolymer materials interactions represents then a major issue for the pharmaceutical industry in the delivery of drugs.

A number of parameters have been identified to affect the sorption: the physicochemical properties of the drug itself (lipophilicity, pKa, isoelectric point, steric hindrance, concentration), the excipient composition, infusing process (flowrate, medical devices length), the physicochemical properties and chemical nature of the polymer material ${ }^{2-4,11}$ and the nature and amount of plasticizers. A number of experimental procedures ${ }^{2,3,11-16}$ have been developed to determine the drug concentration before/after passing through the medical devices by using HPLC methods to quantify the difference in drug quantities. Experimental procedures focusing on material analyses have also been investigated as an alternative approach but they have not been yet able to completely describe the phenomena involved in drug sorption to medical devices.

Nevertheless, in-depth understanding of sorption obviously requires experiments on the surface state characterization at the interface with the drug solution and drug concentration measurements, but also requires a microscopic description of the sorption with an energetic view. This dual microscopic/energy approach can currently be carried out using molecular simulation methods. ${ }^{17}$ The performance of these theoretical methods is partly based on the quality of the force fields ${ }^{17}$ used to model the interactions between the different species. One can even hope in the near future to design medical devices using the knowledge of the interactions between the different molecular assemblies that are the polymer surface, plasticizer, drug and excipients. In addition, the description of the sorption at the atomistic scale will be of addedvalue for interpreting experiments.

We propose here to investigate the adsorption of two drugs (diazepam and paracetamol) (see Figure 1) on PE and PVC materials. Diazepam is known to interact with medical tubings ${ }^{16,18}$ whereas the paracetamol has not shown any significant adsorption. ${ }^{4,15}$ We aim to model the adsorption process of drug on materials by calculating the potential of mean force $(\mathrm{PMF})$ along the direction normal to the surface. We established the free energy profile of the drug's approach to the material. We completed this energetic characterization of the adsorption process by investigating structural and dynamics properties of the drug such as the adsorption sites, hydration, orientation and diffusion. Along with molecular simulations, we also performed experimental studies in which paracetamol and diazepam were put in contact with tubings made of PE and PVC without additives. Actually, in this study, we decided to not consider the plasticizer and the excipients in order to establish the driving forces of the sorption between the drugs and the polymeric surfaces. The effects of the plasticizer and the excipients will be investigated later and will require additional methodological developments. Few theoretical studies ${ }^{19}$ aimed at simulating the sorption process of drugs into polymeric surfaces have been conducted in the past whereas the PMF calculations have been successfully applied to association, ${ }^{20-22}$ adsorption ${ }^{23,24}$ and exfoliation ${ }^{25}$ processes in comparison with experiments.

The paper is organized as follows. Section 2 describes the methodological aspects of experiments and molecular simulations. In Section 3 we discuss the main results of this work in terms of free energy of adsorption, description of the interfacial region and drug diffusion. Section 4 contains our main conclusions and draws up the perspectives related to this work. 


\section{Materials and methods}

\subsection{Experimental studies}

Experimental sorption studies were conducted to evaluate paracetamol and diazepam concentration variations after static contact with $\mathrm{PE}$ or PVC tubes.

\subsubsection{Materials}

The tubings used in this study are described in Table 1. All the tubings were composed of $\mathrm{PE}$ or PVC without additives and will be henceforth referred to as pure $\mathrm{PE}$ and pure PVC. They were made to order by CAIR LGL (Lisieux, France). $60 \mathrm{~mL}$ polypropylene syringes (Pentaferta, Italy, Ref 002022970) were also used.

The following API were used :

- Paracetamol, purity $>99 \%$, pharmaceutical raw material (Ref 1547095, Cooper Pharmaceutique, Melun, France)

- Diazepam, purity $>99 \%$, chemistry grade (Ref D08988-1G, Sigma-Aldrich Chimie SARL, Saint Quentin Fallavier, France)

\subsubsection{Reagents}

All the reagents used for the chromatographic analysis were of HPLC grade. The following reagents were used : acetonitrile $(\mathrm{ACN})$ 99\% purity (Fisher Chemical, United Kingdom); methanol $99 \%$ purity (Fisher Chemical, United Kingdom); formic acid 98\% purity (Fluka, Germany); monobasic potassium phosphate (Sigma-Aldrich, Germany).

\subsubsection{Samples preparation}

Molarity of paracetamol and diazepam was standardized. Samples were diluted with sterile water to $0.07 \mathrm{mM}$, corresponding to $10 \mu \mathrm{g} \mathrm{mL}^{-1}$ and $20 \mu \mathrm{g} \mathrm{mL}^{-1}$. To obtain complete dissolution, diazepam solutions were stirred at room temperature for $72 \mathrm{~h}$.

\subsubsection{Study design}

Each tubing was first rinsed with the API solution, at a flowrate of $1200 \mathrm{~mL} / \mathrm{h}$ regulated by an electric syringe pump. The rinsing volume of PE and PVC tubings was respectively of $6.9 \mathrm{~mL}$ and $5.3 \mathrm{~mL}$ corresponding to the dead space of each tube. After rinsing, the tubes were completely filled with the API solution, closed at both extremities by a Luer-Lock stopper, and then stored in a climatic chamber (Binder, model KBF240, Gmbh Tuttligen, Germany) for 14 days. Initial API concentration was quantified right after the filling syringe, then evolution of the concentration was assessed at the beginning of the infusion (T0), at day 1 (T1), 2 (T2), 4 (T4), (T7) and 14 (T14). For each analytical time, five independent tubings were used $(n=5)$ and were fully emptied to collect samples. A total of 25 tubings was used for each condition. Quantification was performed with a liquid chromatography system (LC2010-Shimadzu) equipped with an UV detector. Analytical methods and validation data of each API are presented in previously published work. ${ }^{4}$

\subsection{Computational procedures}

The diazepam, paracetamol molecules and the atoms of the PE and PVC surfaces were modeled by using the All-Atom General Amber force field $(\mathrm{GAFF}) .{ }^{26}$ The atomic partial charges were calculated at the B3LYP/6$31++\mathrm{g}(\mathrm{d}, \mathrm{p})$ level $^{27,28}$ using the Cioslowki's atomic polar tensor (APT) method. ${ }^{29-31}$ These calculations were carried out using Gaussian $16^{32}$ package. The water molecules and intermolecular interactions were described by using the TIP4P2005 model. ${ }^{33}$

The molecular dynamics simulations were performed with the LAMMPS package. ${ }^{34}$ The integration of the equations of motion was performed with the standard velocity-Verlet algorithm by using a timestep of $2 \mathrm{fs}$. The SHAKE algorithm ${ }^{35}$ was used to constrain all the $\mathrm{H}$-based bonds and the $\mathrm{HOH}$ angle of water molecules. The Lennard-Jones crossing parameters were calculated using Lorentz-Berthelot 
Table 1: Description of the tubings and syringes used in this study. All the tubings were composed of PE or PVC without additives.

\begin{tabular}{llccc}
\hline Device & Reference & Material & $\begin{array}{c}\text { Length } \\
(\mathrm{cm})\end{array}$ & $\begin{array}{c}\text { Inner diameter } \\
(\mathrm{mm})\end{array}$ \\
\hline Tubing & Made to order & pure PE & 55 & 4 \\
Tubing & Made to order & pure PVC & 42 & 4
\end{tabular}

rules (i.e $\epsilon_{i j}=\sqrt{\epsilon_{i i} \epsilon_{j j}}$ and $\sigma_{i j}=\frac{\sigma_{i i}+\sigma_{j j}}{2}$ where $i$ and $j$ refer to the force centers and $\epsilon$ and $\sigma$ are the energy parameter and diameter of atoms of types $i$ and $j$, respectively). The NoseHoover ${ }^{36,37}$ thermostat and barostat algorithm was used to maintain the temperature at $300 \mathrm{~K}$ and the pressure at $1 \mathrm{~atm}$. The cutoff radius was fixed to $12 \AA$ for the repulsion-dispersion interactions. The periodic boundaries conditions were applied in the three directions. The electrostatic interactions were handled with the PPPM 3D method. ${ }^{38,39}$

In semi-crystalline PE, both crystalline and amorphous states coexist. In crystalline regions, the polymer chains are highly ordered and the amorphous region chains adopt a random coil conformation. In order to simplify the simulation and to have an impact of the surface state on the adsorption, the amorphous and crystalline states will be considered independently. For the cristalline PE surface, we chose to model two surface states simulating two different approaches.

The crystalline PE surface is formed by 70 chains of 20 monomers or 40 carbon and 80 hydrogen atoms each. All chains are in the all-trans conformation, their main axis aligned with the $y$-axis. Chains are arranged according to the polyethylene orthorhombic structure with Pnam space group and unit cell parame$\operatorname{ters}^{40}(a=7.40 \AA, b=4.93 \AA, c=2.53 \AA)$. The resulting $\mathrm{PE}$ slab consists of $7 \times 5 \times 24$ unit cells. Two types of interacting planes are considered : the (010) and (100) planes. When the water molecules are facing the (100) plane, the solidliquid interface is expected to be smoother than with the (010) plane (see Figure S1 of the Sup- porting Information). The reader is redirected to The Supporting Information for illustration of the different geometries of the PE and PVC surfaces. The crystalline surfaces were relaxed by performing consecutive molecular dynamics simulations in the constant-NVT and constantNPT ensembles where $N, V, T$ and $P$ mean the number of molecules, volume, temperature and pression, respectively. The final density was about $1.02 \mathrm{~g} \mathrm{~cm}^{-3}$.

For amorphous PE, 14 chains of 100 monomers each were constructed step by step in order to propose random equilibrium chain configurations. The resulting configuration was relaxed in successive simulations in the NPT ensemble at different increasing temperatures up to $600 \mathrm{~K}$. The final configuration is then obtained after NVT runs at $300 \mathrm{~K}$. The resulting density was found to converge to $0.87 \mathrm{~g} \mathrm{~cm}^{-3}$. As PVC is an atactic and basically amorphous, we modeled the PVC surface with 9 chains of 95 monomers each. All the chains in this study were atactic. After repeated heating-cooling cycles in NPT simulations and NVT simulations at $300 \mathrm{~K}$, the final density was found to $1.37 \mathrm{~g} \mathrm{~cm}^{-3}$.

Classical molecular dynamics simulations were performed on a system composed of a polymeric surface (PE or PVC), a drug (diazepam or paracetamol) and 4000 water molecules. The equilibration period was fixed to 500 ps and the averages of the structural and thermodynamic properties were calculated over a period of $40 \mathrm{~ns}$ during the acquisition phase (see Figure 1c for a representation of the system model).

The potential of mean force (PMF) was 
calculated by using an extended version $(\mathrm{eABF})^{41-43}$ of the Adaptive Biasing Force (ABF) method. ${ }^{44-47}$ eABF takes the route of improving the sampling of the configurational space by adding a fictitious degree of freedom and define an extended potential. The correct free energy profile was then recovered by using the corrected z-averaged restraint (CZAR) estimator. ${ }^{42} \mathrm{~A}$ brief description of the calculation of the derivative of the Gibbs free energy profile with eABF is given in the Supporting Information along with the values of the parameters used for the spring constant and fictitious mass. The calculations were performed in the $\mathrm{N} p_{z z}$ AT statistical ensemble where the surface area $A=L_{x} L_{y}$ is constant. In this ensemble, the PMF profile corresponds to the Gibbs free energy profile $G(z)$ along the $z$-coordinate between the $z$-positions of the center of mass of the polymer surface and drug molecule. The maximum value of the reaction coordinate was set to $28 \AA$. The region of the polymer surface allowed to be sampled corresponded to an area of $64 \AA^{2}$ around the average position $\left(x_{f}, y_{f}\right)$ of the drug calculated during a standard molecular dynamic simulation. Accordingly, the $x$ and $y$ transition coordinates were constrained on the domains $\left[x_{f}-4 ; x_{f}+4\right]$ and $\left[y_{f}-4 ; y_{f}+4\right]$, respectively. A number of independent PMF calculations (between 5 and 9) were performed by moving the drug $\left(x_{f}, y_{f}\right)$ on different regions of the surface in order to estimate the statistical fluctuations of the PMF curves. For amorphous surfaces, the shape of the positive part of the PMF curve can change with respect to the roughness of the surface at the atomistic level. However, the values of the Gibbs free energy minimum remained unchanged within the standard deviations.

We adopted the convention that the Gibbs free energy $G(z)$ is zero for the largest separation distance between the drug molecule and the polymeric surface. Statistical fluctuations on the Gibbs free energy profiles were calculated by performing 10 independent calculations of $G(z)$ with different initial configurations as reported in the Supporting Information. The standard deviations are estimated to be in the range of $2-3 \mathrm{~kJ} \mathrm{~mol}^{-1}$ in the region of the Gibbs free energy minimum. They can be smaller at larger separation distances and much larger at smaller separations (see Figure S2 of the Supporting Information).
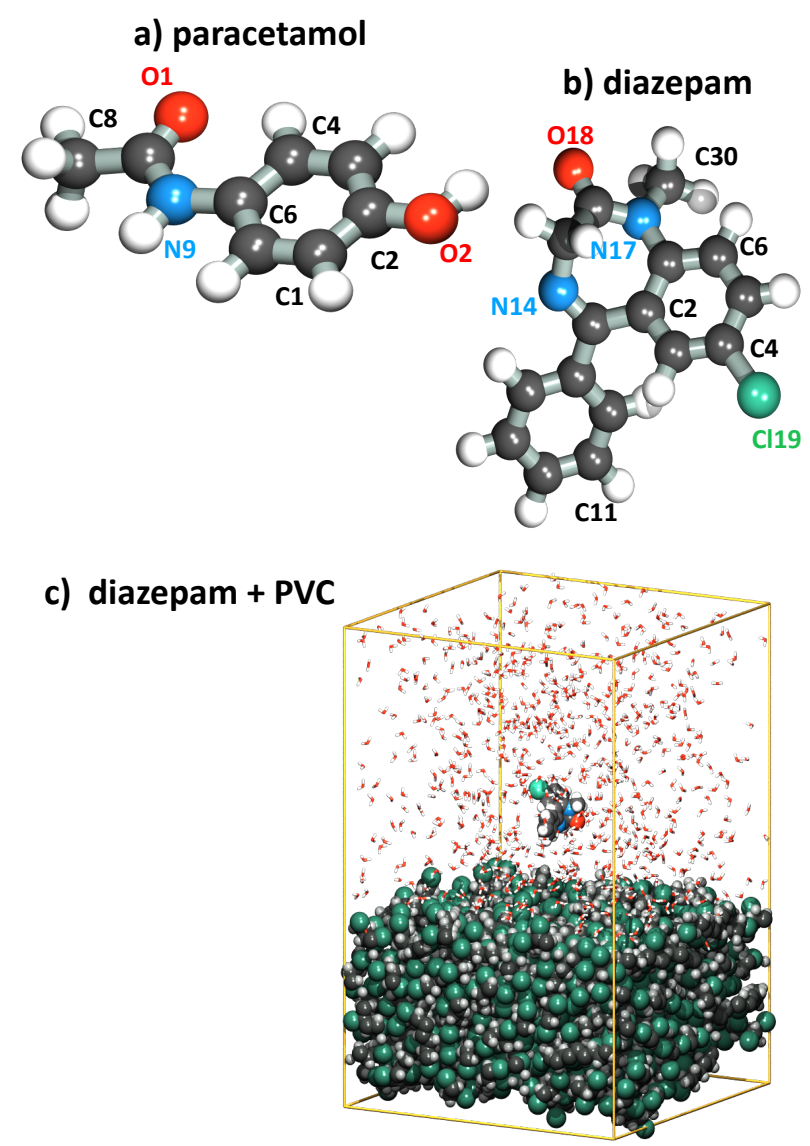

Figure 1: Conformations of (a) paracetamol and (b) diazepam molecules and c) a diazepam molecule adsorbed onto a PVC surface.

\section{Results and discussion}

Figure 2 shows the profiles of the Gibbs free energy as a function of the $z$-separation distance between the centers of mass of the surface and the drug molecules. For all the studied cases, the adsorption is thermodynamically favored : all the PMF curves show a negative minimum of $\Delta G$ close to the surface. The values of the free energy minimum fall into a range of -35 to $-15 \mathrm{~kJ} \mathrm{~mol}^{-1}$ (see Table 2). This order of magnitude indicates that the adsorption of these drugs molecules can be attributed to a pure physical adsorption through van der 
Waals interactions, as expected with a classical description of the interactions of models used. The Gibbs free energies of adsorption calculated here are in the same range of ones measured from sorption isotherms of drugs on biopolymer/magnetite composites. ${ }^{48}$ For both drugs, we do not see any major differences in the heats of adsorption and shapes of the PMF profiles between the (100) and (010) faces of crystalline $\mathrm{PE}$ and amorphous PE. A larger well in the PMF profile can be identified with an amorphous surface for the paracetamol as shown in Figure 2a.
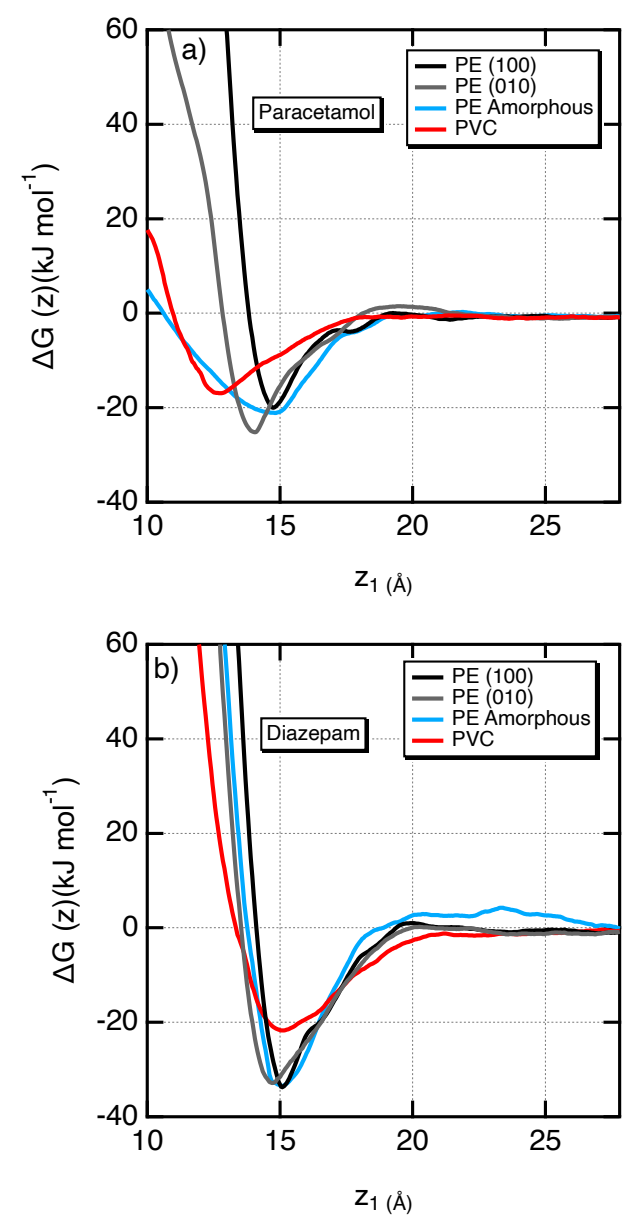

Figure 2: Gibbs free energy profiles $\Delta G\left(z_{1}\right)$ of the interaction of a) paracetamol and b) diazepam drugs with different surface geometries. $z_{1}$ is defined as the distance between the centers of mass of the drug and surface.

We used the HPLC method after static contact to detect any loss by sorption, and so to assess whether there is an interaction of the paracetamol and diazepam solutions with PE and

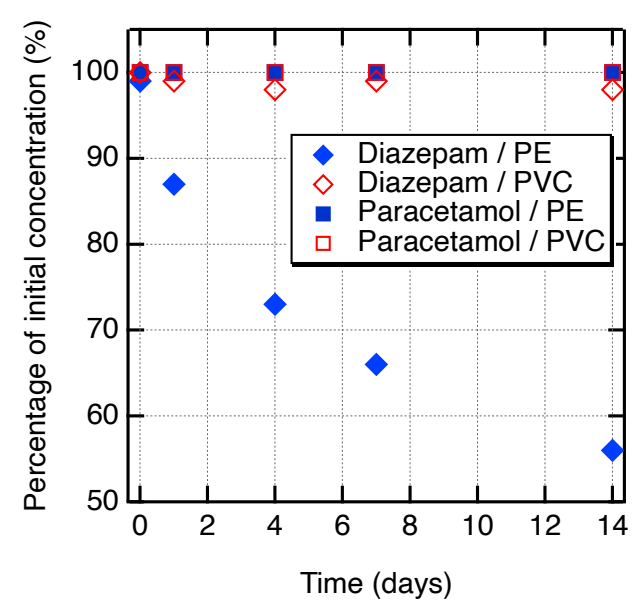

Figure 3: Evolution of paracetamol and diazepam concentrations in static contact with PVC and PE tubings, compared to initial concentrations. The error bars on the concentrations values are less than $1 \%$ and cannot be read with the scale used.

PVC. Figure 3 shows the evolution over time (in days) of the concentration of both drugs during static contact with PVC and PE tubings. For both materials, the paracetamol solution remained unchanged for 14 days. Diazepam concentration was also stable for 14 days in contact with PVC tubing, but when in contact with PE tubing, a drug loss was observed from day 1 and fell to $56 \%$ of the initial concentration at 14 days. According to Table 2 , the Gibbs free energy of adsorption is minimum for the system diazepam/PE (around -33 $\mathrm{kJ} \mathrm{mol}^{-1}$ ), and the physisorption is more favorable in this case. When comparing experimental results to simulations, it appears that no drug loss by sorption was observed when Gibbs free energy was comprised between 0 and $-25 \mathrm{~kJ}$ $\mathrm{mol}^{-1}$, which corresponds to a weak adsorption. Yet, weak adsorption could have led to a slight drug loss, hardly detectable with our analytical procedure. From this combined approach, we can establish a threshold value of around -30 $\mathrm{kJ} \mathrm{mol}^{-1}$ above which we did not observe any adsorption on PVC and PE tubings.

The PMF calculations give not only the depth of the adsorption wells, but also can provide informations through their shapes about the adsorption process. The full PMF curves can then be used to estimate the number of ad- 
Table 2: Gibbs free energy of adsorption $\left(\mathrm{kJ} \mathrm{mol}^{-1}\right)$ obtained from the minimum of $\Delta G\left(z_{1}\right)$. The statistical fluctuations are within $2-3 \mathrm{~kJ} \mathrm{~mol}^{-1}$ for the Gibbs free energy minimum (see Figure S2 of the Supporting Information) and within about $10 \%$ for the adsorption length $l_{\text {ads }}$

\begin{tabular}{lcccc}
\hline Surface & $\begin{array}{c}\Delta G_{\mathrm{ads}}^{o} \\
\left(\mathrm{~kJ} \mathrm{~mol}{ }^{-1}\right)\end{array}$ & $\begin{array}{c}l_{\mathrm{ads}} \\
(\mu m)\end{array}$ & $\begin{array}{c}\Delta G_{\mathrm{ads}}^{o} \\
\left(\mathrm{~kJ} \mathrm{~mol}^{-1}\right)\end{array}$ & $\begin{array}{c}l_{\mathrm{ads}} \\
(\mu m)\end{array}$ \\
\hline paracetamol & \multicolumn{2}{c}{ diazepam } \\
PE (100) & -20.0 & 0.2 & -33.7 & 36.0 \\
PE (Amorphous) & -25.2 & 1.5 & -32.7 & 38.0 \\
PVC & -20.9 & 0.8 & -33.4 & 56.0 \\
& -16.8 & 0.1 & -21.5 & 0.8
\end{tabular}

sorbed molecules. The number of adsorbed drug molecules per unit area, at equilibrium is the same as the number of such molecules in a slab of solution of thickness $l_{\mathrm{ads}}$, and can be calculated as

$$
l_{\text {ads }}=\int_{\text {surface }}^{\infty}\left(\exp \left(-\frac{\Delta G(z)}{R T}\right)-1\right) \mathrm{d} z
$$

Adsorption has a significant impact on the concentration in the solution if $l_{\text {ads }}$ is not negligible versus the volume/area ratio of the container, taking into account that roughness actually increases the effective area. Equation (1) neglects the possibility of absorption and the interactions between drug molecules. The predicted impact on the concentration is underestimated. Nevertheless, the estimated $l_{\text {ads }}$ given in Table 2 strongly correlates with the experimental decrease of concentration of diazepam when it is contact with $\mathrm{PE}$ tubing.

Figure 4a compares the PMF curves between paracetamol and diazepam in water. In the case of the PE surface, we can conclude that the physisorption is more favorable with diazepam than with paracetamol with an energy benefit of at most $14 \mathrm{~kJ} \mathrm{~mol}^{-1}$. This is also true with PVC but the energy gain is less, it is only about $5 \mathrm{~kJ} \mathrm{~mol}^{-1}$. The physisorption is weaker with PVC than with $\mathrm{PE}$ with a more pronounced difference with diazepam than with paracetamol. The position of the free energy minimum is shifted to higher separation distances with diazepam which could be explained by the fact that its van der Waals volume is 1.8 times greater than that of paracetamol. Typical snapshots are shown at different separation distances in Figure S3 of the Supporting Information.

The adsorption phenomenon is a combination of two processes: the adsorption of the drug molecule and the desorption of water molecules initially adsorbed at the polymeric surfaces. These two contributions are part of the calculated $\Delta G(z)$ reported in Figures 2 and 4 a. To better understand the adsorption process in terms of energetic contributions, we also investigated both the drug adsorption without any water molecules and the adsorption of a water molecule in water and gas phase. Figure (4a) shows that the interaction of a water molecule with the polymer materials (PE and PVC) is very weakly thermodynamically favored with respect to its interaction with water molecules in bulk water phase (represented here by the largest values of $z_{1}$ ). Actually, with the crystalline (010) face, we dot not observe a marked free energy minimum but rather a region of negative value around $-0.4 \mathrm{~kJ} \mathrm{~mol}^{-1}$ to be moderated with respect to statistical fluctuations (see Figure S2a). In the case of the interaction of the water with amorphous PE surface, a flat region of free energy minimum of $-2.0 \mathrm{~kJ}$ $\mathrm{mol}^{-1}$ occurs at a distance to the surface corresponding to about the diameter of the oxygen atom of water molecule. In gas phase (see Figure $4 \mathrm{~b}$ ), the PMF of the adsorption of a water molecule on PE shows a minimum at $-3.6 \mathrm{~kJ}$ $\mathrm{mol}^{-1}$ and $-8 \mathrm{~kJ} \mathrm{~mol}^{-1}$ for the adsorption on 

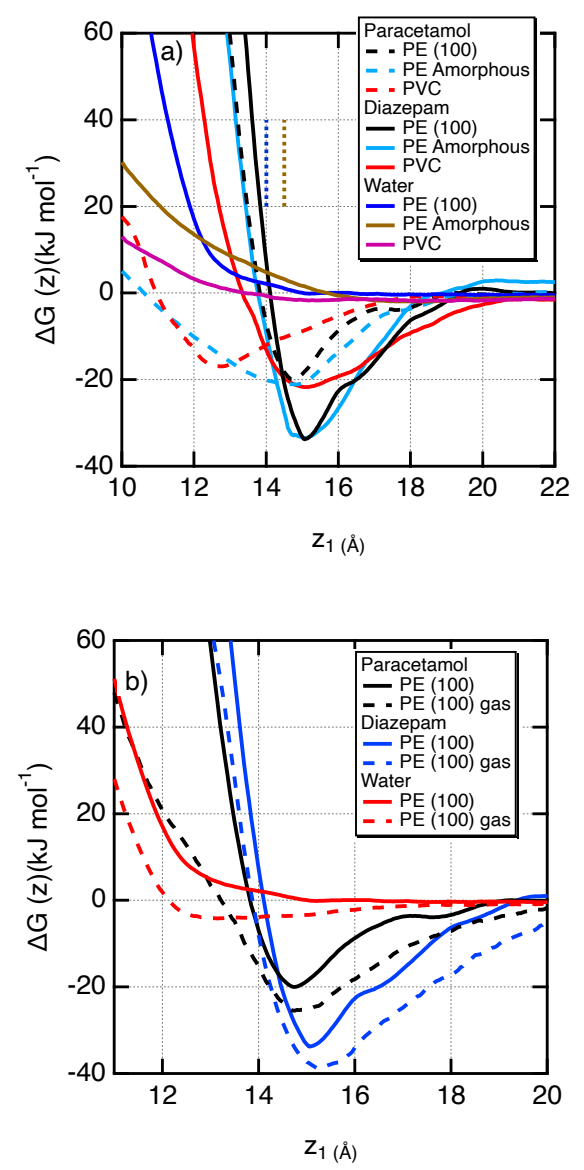

Figure 4: Comparison of Gibbs free energy profiles $\Delta G\left(z_{1}\right)$ of paracetamol and diazepam in a) water and b) gas state. The well-depths $\left(\Delta G_{\text {ads }}^{o}\right)$ are then $-25.4,-38.8$ and $-3.6 \mathrm{~kJ} \mathrm{~mol}^{-1}$ for paracetamol, diazepam and water in gas phase, respectively. We also give for completeness the PMF curves corresponding to the adsorption process of a water molecule on PE and PVC materials in water and on PE in gas phase. The vertical dotted lines in a) shows the positions of the crystalline and amorphous PE surface.

PVC (PMF curve not shown here). These very small values of adsorption in gas phase explain why the adsorption of a water molecule in water is not favored. Indeed, the energetic gain calculated in gas phase is counterbalanced by the loss of favorable intermolecular hydrogen bond interactions when the water molecule approaches the surface. From these PMF calculations for water, we deduce that the desorption of water molecules does not make any favorable contributions to the adsorption of the drug molecule.
In addition, in the gas phase, the PMF profiles involving the adsorption of drug molecules indicates that the well-depth of the Gibbs free energy curves increases by -5.4 and $-5.1 \mathrm{~kJ} \mathrm{~mol}^{-1}$ with respect to the adsorption in water with paracetamol and diazepam molecules, respectively. This indicates that the net interaction between the polymeric surfaces and these drug molecules are favorable. The difference between the PMFs in the gas phase and water are then explained by an energetic cost due to possible favorable interactions of each drug with water.

The orientation of the drug molecule with respect to the surface is characterized by the angle $\theta$ between two specific vectors of the molecule (see caption of Figure 5). If $\theta$ is either $0^{\circ}$ or $180^{\circ}$, it means that the molecule flattens out on the surface with a maximum number of interacting atoms. It is interesting to observe that the biased PMF simulations show that all the orientations of the molecule observed at the largest separation distances remain still possible when it is interacting with the surface. The drug molecule can turn completely on itself on the surface of the polymer. Only a region of separation distances between 15 and $20 \AA$ contrains the molecule and limits degree of freedoms related to its orientation with respect to the surface. The orientational restriction in this zone is much more pronounced with diazepam because of its size. Nevertheless, even with diazepam, the PMF calculations show that the adsorption of the drug on the surface does not prevent it to cover a large spectrum of orientations that are then all equivalent from a free enthalpy viewpoint. As shown in Figure 5a, the standard molecular simulations with paracetamol confirms this point by reproducing the different possible orientations of the drug whereas the same molecular simulations with diazepam trap the molecule in a smaller region of possible orientations (see Figure 5b) during the simulation. It is then needed to change the initial orientation of diazepam to sample the other region. This is in line with a stronger interaction of the diazepam with the PE surface. This also shows that the PMF simulations perform very well in the sampling of all the degree of freedoms. 

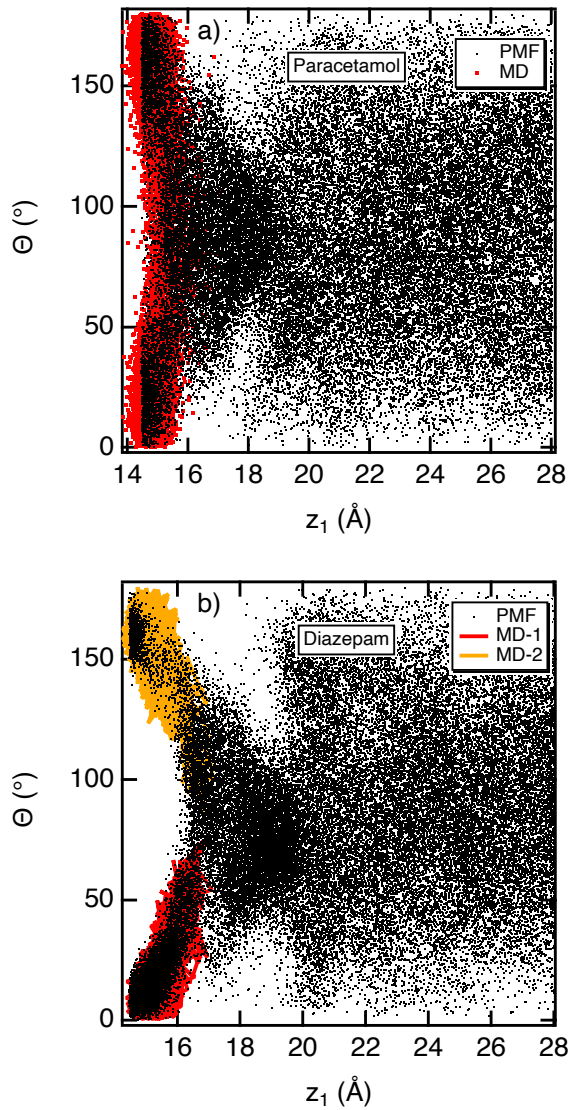

Figure 5: Distributions of the angle $(\theta)$ between the vectors formed from the atoms $\mathrm{C} 2$ and $\mathrm{C} 4$ on the one hand and $\mathrm{C} 4$ and $\mathrm{C} 6$ on the other for a) paracetamol and b) diazepam drugs. See Figure 1 to see the positions of the atoms in the drug molecules. These distributions are calculated with the (100) plane of PE. The black points correspond to the values of $\theta$ calculated in the configurations of the PMF simulations whereas the red and orange points represent the angles calculated in standard molecular dynamics simulations. In b) the two standard molecular dynamics simulations use two different initial configurations in the value of $\theta$.

We will focus on the description of the polymer-water interface by calculating the atomic density profiles along the $z$-direction from standard molecular simulations. We show in Figure S4 of the Supporting Information that these standard molecular simulations sample the region of the free energy minimum establishing that all the properties calculated afterwards correspond to those of the most thermodynamically stable configurations. The atomic density profiles of the atoms of the surface, water and drug molecules are shown in Figure 6 for different surface models. These profiles highlight that the polymer-water interface is radically different with an amorphous compared to a crystalline surface. With crystalline surfaces, the interface is characterized by adsorption peaks whereas the density profile of water progressively decrease in the interfacial region with amorphous surfaces. For crystalline surfaces, the drug molecules adsorb onto the surfaces with adsorption peaks that coincide with those of water molecules. For amorphous surfaces, the drugs can penetrate deeper than water into the material due to the local roughness but they remain in contact with both polymer and water molecules, thus leading to an adsorption and not an absorption. We also observe a slight difference in the density profiles between the (100) and (010) PE faces. Actually, the (010) face increases the size of the interaction zone into the material with the drug and slightly smoothes the density profiles. To summarize, Figure 6 establishes an adsorption through a structural analysis in all cases even if the experiments developed did not show any show drug loss for the paracetamol solution. Only the calculation of the Gibbs free energy of adsorption establishes a weaker adsorption for paracetamol/PE comparatively to diazepam/PE systems.

First, we focused on the local arrangements of paracetamol at the polymer surface. For the PE (100) face, the peaks of the density profiles are almost all located at the same $z$-position and the interaction zone extends over about $5 \AA$ in the $z$-direction. This indicates that paracetamol is positioned itself to be flat as possible on the surface. For the PE (010) face, (see Figure $\mathrm{S} 5 \mathrm{~b}$ in the supporting Information), the profiles are widening with small shift of the $z$-positions of oxygen atoms towards the water. This is in line a slightly less smooth interacting surface at a molecular scale. As expected, the broadening of the density profile becomes more pronounced with amorphous surfaces. Indeed, the interface between paracetamol and surfaces extends over $8 \AA$ for the PE and PVC surfaces. In addition, with PVC, we observe a larger shift of the pro- 


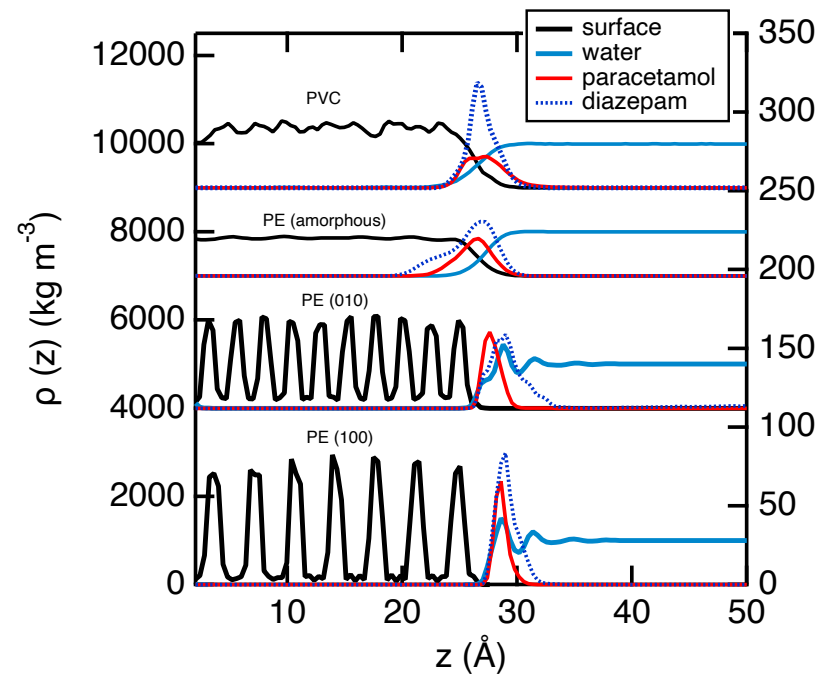

Figure 6: Atomic density profiles of the PE and PVC surfaces and water molecules (left axis). The atomic density profiles of the paracetamol and diazepam molecules are calculated by excluding the hydrogen atoms and must be read on the right axis. For PE (100), PE (amorphous) and PVC systems, the density profiles of the atoms of the surface and water molecules are offset by 4000,7000 and $9000 \mathrm{~kg} \mathrm{~m}^{-3}$ on the left axis whereas the density profiles of the drug molecules are offset by 112, 196 and 252 $\mathrm{kg} \mathrm{m}^{-3}$ on the right axis, respectively.

files of the oxygen atoms towards water. The fact that oxygen atoms point towards the water phase implies that the nitrogen atom points are rather located at the surface of the material. The calculation of the hydrogen bonds between paracetamol and water molecules is based upon the following definition : the X$\mathrm{H}$..O distance is required to be less than $2.5 \AA$ and the $\mathrm{H}-\mathrm{X} . . \mathrm{O}$ angle to be less than $30^{\circ}$ where $\mathrm{X}$ represents either $\mathrm{O}$ or $\mathrm{N}$. The number of hydrogen bonds between paracetamol and water molecules changes from 2.5 to 2.8 as a function of the nature of the surface whereas the paracetamol molecules gives 3.4 hydrogen bonds in bulk water phase.

Secondly, we turn our attention to the atomic density profiles of diazepam interacting with the same surfaces. The density profiles are given in Figures 7 c), d) and f). As underlined in Figure 5b, we calculated the atomic density profiles over two molecular simulations that sample two different orientations of diazepam. The density profiles corresponding to the second sets of possible orientations are given in Figure S5 b), c) and d) of the Supporting Information. As expected from the size of the molecule, we observe that the atomic profiles are slightly more distributed in the $z$-direction with an interaction zone covering about $7 \AA$. In a firt orientation, diazepam's oxygen atoms distribute themselves near the water molecules whereas the chlorine atom is close to the atoms of the PE (100) surface. The second orientation of diazepam shows a density profile of the oxygen less shifted towards the water phase. These changes in the density profiles between the two orientations do not lead to radically different conformations of the molecule on the surface. For the amorphous surfaces, the overlap region between the diazepam and surfaces extends over $12 \AA$ for PE and $8 \AA$ for PVC. In all the profiles, these are the chlorine and carbon (C11 and C40) that preferentially interacts with the polymer material. With PVC, we observe that a large part of hydrophilic atoms remain in the PVC-water interface therefore limiting to a strict minimum the number of hydrogen bonds with the water molecules. Indeed, whereas diazepam gives 2.2 hydrogen bonds with water, the interaction with PVC reduces by 0.1 its ability to form hydrogen bonds and by 0.2 with amorphous PE. The radial distribution functions between drugs and water molecules in bulk water, given in Figure S6 of the Supporting Information, show that these two molecules are rather considered as lipophilic in line with their octanol-water partition coefficients. ${ }^{4}(\log P=$ 3.1 and 0.9 for diazepam and paracetamol, respectively).

Another factor that can inform us about the nature of the adsorption of the drug is the diffusion coefficient estimated in the $x, y$ and $z$ directions. We calculate this property on the three directions by using the expression given in the following equation

$$
D_{\alpha \alpha}=\frac{1}{2} \lim _{t \rightarrow \infty} \frac{d}{d t}\left\langle\left[r_{\alpha}(t)-r_{\alpha}(0)\right]^{2}\right\rangle
$$

where $r_{\alpha}(t)$ is the position of the center of mass 

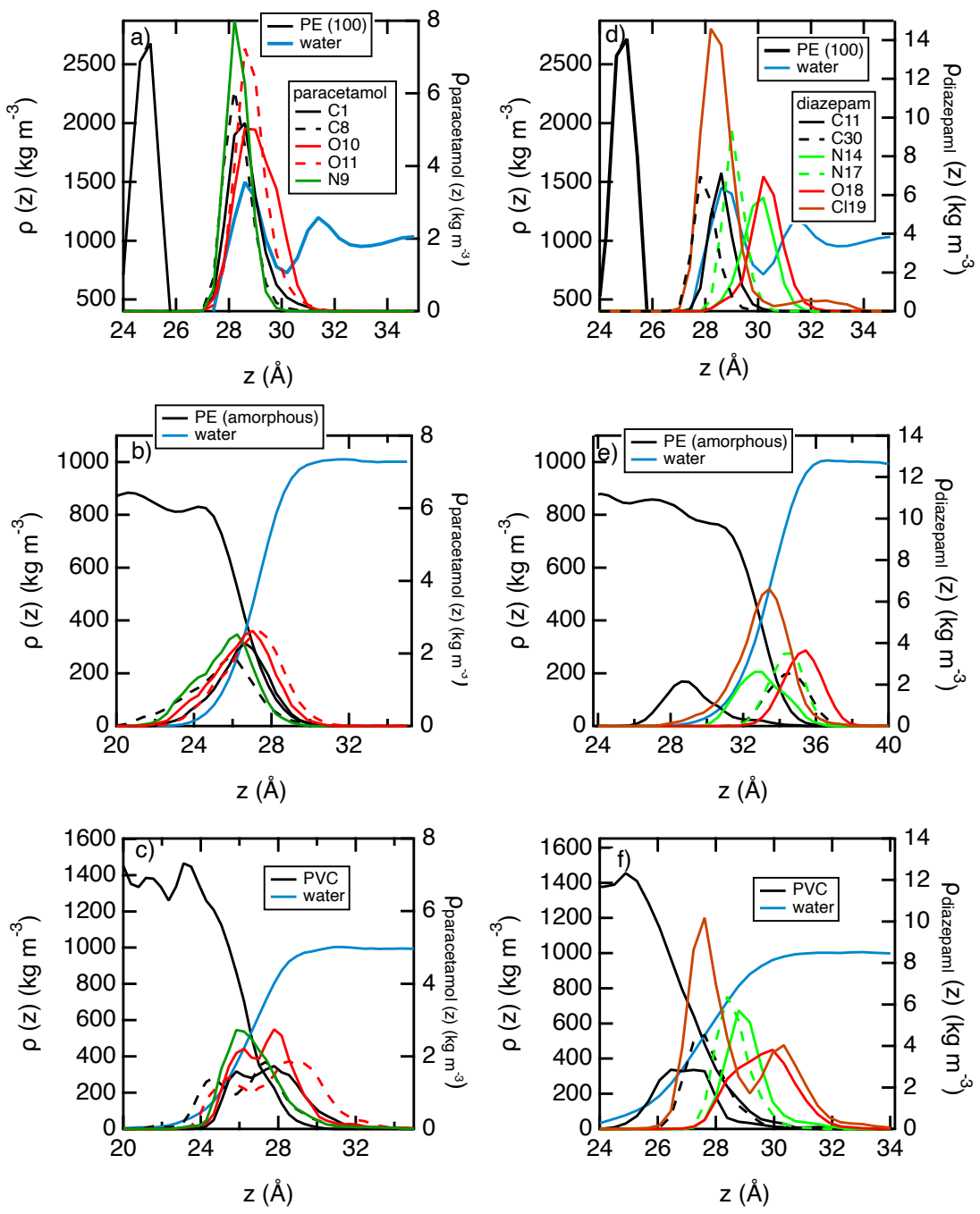

Figure 7: Atomic density profiles of some atoms of the drug molecules as indicated in the legend (right axis). On the left axis, are represented the atomic density profiles of the atoms of water molecules and polymer material. a) b) and c) corresponds to the profiles of paracetamol in interaction with the crystalline PE (100) plane, amorphous PE and PVC surfaces, respectively. d), e) and f) parts corresponds to the same surfaces interacting with diazepam.

of the drug molecule at time $t . t=0$ refers to the time origin. The average is performed over different time origins. The diffusion coefficient $^{49} D_{\alpha \alpha}$ is then calculated as one-half of the slope of $\left\langle\left[r_{\alpha}(t)-r_{\alpha}(0)\right]^{2}\right\rangle$ versus time. The reader is redirected to Figure S7 in the Supporting Information to see the representation of the mean square displacements in a linear scale. Figure 8 shows the same mean square displacements in logarithmic scales. We reported for comparison the mean square displacements of water in bulk conditions and the slope 1 corresponding to the diffusion regime. For the crys- talline PE surfaces, we observe that the diffusion coefficient of paracetamol is about 125 $\AA^{2} \mathrm{~ns}^{-1}$ in the $y$-direction and ranges from 2 (010 face) to 23 (100 face) $\AA^{2} \mathrm{~ns}^{-1}$ in the $x$ direction whereas no diffusion is observed in the $z$-direction (see also Figure S7 of the Supporting Information). These values of diffusion are in line with structural features of the PE surface at the molecular scale, namely the more important diffusion occurs in the direction of the main axis of the PE chains. For these crystalline surfaces, the diffusion of the drug in the $y$-direction is about half the self-diffusion coefficient of water. For amorphous PE and PVC 

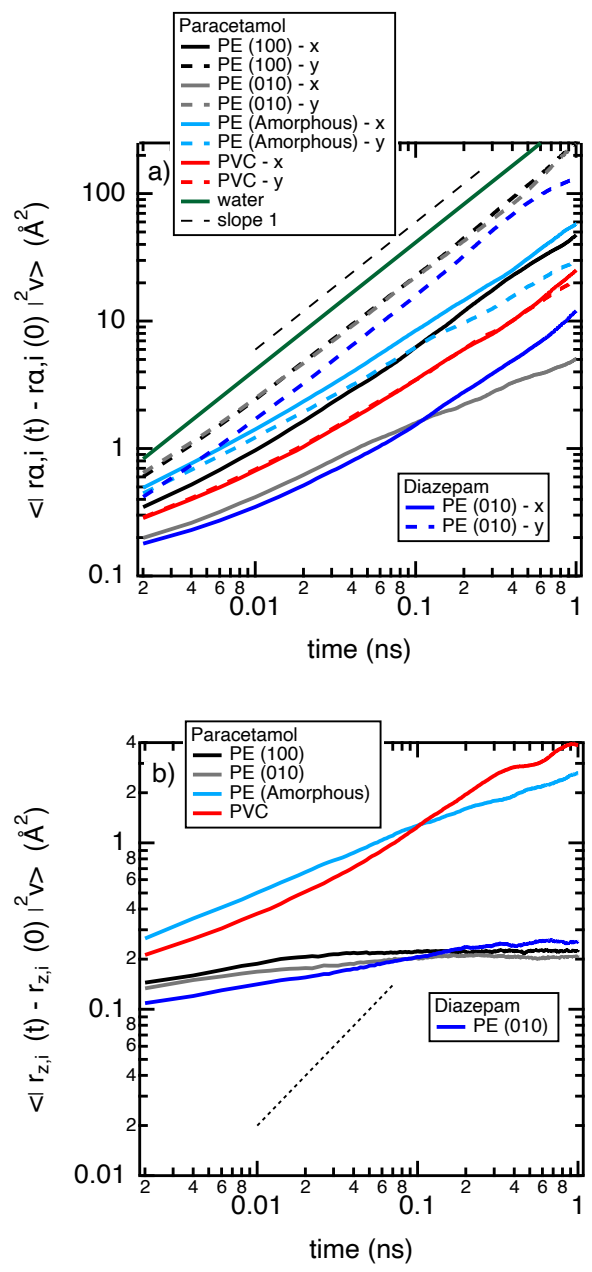

Figure 8: Log-Log graph of the a) $x, y$ and b) z-components of mean square displacements versus time of adsorbed paracetamol and diazepam on different surfaces. We give for comparison the mean square displacement of water molecules in water. The slope 1 represents the diffusion mode occurring at long-time for which the motion is governed by collisions.

surfaces, we observe a slowdown of the diffusion in both $x$ and $y$ directions with diffusion values in 10-28 $\AA^{2} \mathrm{~ns}^{-1}$ and an increase of the diffusion in the $z$-direction (see Figure $8 \mathrm{~b}$ ). For the diazepam, we observe the same behaviors. With the PE (010) interface, its diffusion coefficient is reduced to $70 \AA^{2} \mathrm{~ns}^{-1}$ compared to $125 \AA^{2}$ $\mathrm{ns}^{-1}$ for the paracetamol.

\section{Conclusions}

We report here a combined approach of experiments and molecular simulations to character- ize the sorption of paracetamol and diazepam to pure PVC and PE materials. The simulated Gibbs free energy of adsorption that are within the range of -35 to $-15 \mathrm{~kJ} \mathrm{~mol}^{-1}$ establish a physical adsorption through van der Waals interactions. The most favorable adsorption is predicted with diazepam that adsorbs on PE. The nature of the PE surface (amorphous or crystalline) does not affect the thermodynamics. Very interestingly, the experiments showed that diazepam suffered a $56 \%$ loss in concentrations using static contact with $\mathrm{PE}$ tubings whereas no drug loss was measured for paracetamol for both materials. This coupling between experiments and molecular simulation is able here to provide a threshold value of about $-30 \mathrm{~kJ} \mathrm{~mol}{ }^{-1}$ above which we did not detect any drug loss. This is an essential result that should enable molecular simulations to be used for the prediction of adsorption properties and further for the design of new polymeric surface with specific properties.

Once the adsorption was characterized from a thermodynamic point of view, we tried to describe the polymer-drug-water region at the atomistic level. The adsorption of drugs on $\mathrm{PVC}$ and PE polymer materials is not restrictive in terms of orientations. The shape of the polymer-water interface changes radically between an amorphous and cristalline surface. The water molecules exhibit adsorption peaks with cristalline surfaces whereas the interface that shows an overlap between polymer chains and water is wider with amorphous polymer surfaces. The adsorption of diazepam and paracetamol is analyzed through local atomic positions. Theses analyses indicate the drug is flattened on the surface thus favoring, whenever possible, interactions with water throughout hydrogen bonds. The diffusion of drug molecules on the surface differ significantly between amorphous and cristalline surfaces. With cristalline surfaces, the drug diffuses mainly in the direction of the main axis of the PE chains.

To conclude, the adsorption process of diazepam and paracetamol on PE and PVC materials has been investigated at the energetic, structural and dynamics levels. The comparison with experiments has allowed us to define 
the value of Gibbs free energy of adsorption which can lead to possible drug loss. The structural description of the polymer surface at the molecular is essential for a better understanding of the role of interactions that leads to significant sorption of drugs.

In this study, experiments and simulations have considered PVC materials without plasticizers. Yet, plasticized PVC medical tubings are widely used for the infusion of medications and the presence of plasticizer is also expected to increase adsorption mechanisms. Future work will be to develop more realistic models to model plasticized PVC materials. This type of work will require to develop specific methodologies for an efficient sampling of the plasticizer into the material and at the interface. We can hope to calculate the potential of mean force of the adsorption of the drug onto the plasticized material and evaluate the impact of the plasticizer on the interactions and interfacial properties.

Acknowledgement The authors acknowledge the financial support received from the Auvergne Rhônes Alpes regional council through the program "Pack Ambition Recherche" (MEDSIM-2019). The authors would like to thank colleagues of SimatLab for stimulating discussions about the results. SimatLab is a joint public-private laboratory dedicated to the modeling of polymer materials and supported by Michelin, Clermont Auvergne University (UCA), SIGMA Clermont and CNRS. The authors also thank CAIR LGL for their financial support for the discussion about the MEDSIM project and for providing the tubings used in this study. Computations have been performed on the supercomputer facilities of the Mésocentre Clermont Auvergne University.

\section{Supporting Information Avail- able}

Description of the simulated polymer materials, brief presentation of the extended-ABF technique, typical snapshots resulting from the PMF calculations, atomic density profiles and radial distribution functions are given in the Supporting Information.

\section{References}

(1) Bernard, L.; Décaudin, B.; Lecoeur, M.; Richard, D.; Bourdeaux, D.; Cueff, R.; Sautou, V. Analytical Methods for the Determination of DEHP Plasticizer Alternatives Present in Medical Devices: a Review. Talanta 2014, 129, 39-54.

(2) Jin, S.-E.; You, S.; Jeon, S.; Byon, H.J.; Hwang, S.-J. Evaluation of Drug Sorption to PVC- and Non-PVC-based Tubes in Administration Sets Using a Pump. J. Vis. Exp. 2017, 121, e55086.

(3) Treleano, A.; Wolz, G.; Brandsch, R.; Welle, F. Investigation into the Sorption of Nitroglycerin and Diazepam into PVC Tubes and Alternative Tube Materials during Application. Int. J. Pharm. 2009, 369, 30-37.

(4) Tokhadze, N.; Chennell, P.; Bernard, L.; Lambert, C.; Pereira, B.; MailhotJensen, B.; Sautou, V. Impact of Alternative Materials to Plasticized PVC Infusion Tubings on Drug Sorption and Plasticizer Release. Sci Rep 2019, 9, 18917.

(5) Maiguy-Foinard, A.; Blanchemain, N.; Barthélémy, C.; Décaudin, B.; Odou, P. Influence of a Double-Lumen Extension Tube on Drug Delivery: Examples of Isosorbide Dinitrate and Diazepam. PLoS ONE 2016, 11, e0154917.

(6) Woodward, Z.; Brooks, P.; MorrisSmith, B.; Wallis, M.; Ogbourne, S. M. Adsorption and Leachable Contamination of Flucloxacillin, Cyclosporin and Amiodarone Following Delivery Through an Intravenous Administration Set. Pharm. Res. 2018, 35, 121.

(7) Zahid, N.; Taylor, K. M.; Gill, H.; Maguire, F.; Shulman, R. Adsorption of Insulin onto Infusion Sets Used in Adult Intensive Care Unit and Neonatal Care 
Settings. Diabetes Res. Clin. Pract. 2008, 80, e11-e13.

(8) Hewson, M. P.; Nawadra, V.; Oliver, J.; Odgers, C.; Plummer, J.; Simmer, K. Insulin Infusions in the Neonatal Unit: Delivery Variation due to Adsorption. $J$. Paediatr. Child Health 2000, 36, 216-220.

(9) Jakobsson, T.; Shulman, R.; Gill, H.; Taylor, K. The Impact of Insulin Adsorption onto the Infusion Sets in the Adult Intensive Care Unit. J. Diabetes Sci . Technol. Online 2009, 3, 213-214.

(10) Masse, M.; Maton, M.; Genay, S.; Blanchemain, N.; Barthelemy, C.; Decaudin, B.; Odou, P. In Vitro Assessment of the Influence of Intravenous Extension Set Materials on Insulin Aspart Drug Delivery. PLoS ONE 2018, 13, e0201623.

(11) Jin, S.-E.; Jeond, S.; Byonb, H.-J.; Hwang, S.-J. Evaluation of Tacrolimus Sorption to PVC- and non-PVC-Based Tubes in Administration Sets: Pump Method vs. Drip Method. Int. J. Pharm. 2017, 528, 172-179.

(12) Jenke, D. R. Evaluation of Model Solvent Systems for Assessing the Accumulation of Container Extractables in Drug Formulations. Int. J. Pharm. 2001, 224, 51-60.

(13) Genay, S.; Luciani, C.; Décaudin, B.; Kambia, N.; Dine, T.; Azarouald, N.; Martino, P. D.; Barthélémy, C.; Odou, P. Experimental Study on Infusion Devices Containing Polyvinyl Chloride: To What Extent are They di(2ethylhexyl)phthalate-free? Int. J. Pharm. 2011, 412, 47-51.

(14) Hacker, C.; Verbeek, M.; Schneider, H.; Steimer, W. Falsely Elevated Cyclosporin and Tacrolimus Concentrations over Prolonged Periods of Time Due to Reversible Adsorption to Central Venous Catheters. Clin. Chim. Acta 2014, 433, 62-68.
(15) Ferchichi, M.; Dhaouadi, H. Sorption of Paracetamol onto Biomaterials. Water Sci. Technol. 2016, 74, 287-294.

(16) Jin, S.-E.; You, S.; Jeon, S.; Hwang, S.J. Diazepam Sorption to PVC- and nonPVC-Based Tubes in Administration Sets with Quantitative Determination using a High-Performance Liquid Chromatographic Method. Int. J. Pharm. 2016, 506, 414-419.

(17) Allen, M. P.; Tildesley, D. J. Computer Simulation of Liquids : Second Edition; Oxford: Clarendon Press, 2017.

(18) Salloum, H. A.; Saunier, J.; AymesChodur, C.; H. Barakat, N. Y. Impact of the Nature and Concentration of Plasticizers on the Ability of PVC to Sorb Drug. Int. J. Pharm. 2015, 496, 664-765.

(19) Feenstra, P.; Brunsteiner, M.; Khinast, J. Prediction of Drug-Packaging Interactions via Molecular Dynamics (MD) Simulations. Int. J. Pharm. 2012, 431, 26-32.

(20) Ghoufi, A.; Bonal, C.; Morel, J. P.; MorelDesrosiers, N.; Malfreyt, P. Structures and Energetics of Complexes of the pSulfonatocalix[4]arene with Ammonium, Alkylammonium, and Tetraalkylammonium Cations in Water Using Molecular Dynamics Simulations. J. Phys. Chem. B 2004, 108, 5095-5104.

(21) Ghoufi, A.; Malfreyt, P. Entropy and Enthalpy Calculations From Perturbation and Integration Thermodynamics Methods Using Molecular Dynamics Simulations: Applications to the Calculation of Hydration and Association Thermodynamic Properties. Mol. Phys. 2006, 104, 2929-2943.

(22) Suh, D.; Jo, S.; Jiang, W.; Chipot, C.; Roux, B. String Method for ProteinProtein Binding Free-Energy Calculations. J. Chem. Theory Comput. 2019, 5, 5829-5844. 
(23) Comer, J.; Chen, R.; Poblete, H.; VergaraJaque, A.; Riviere, J. E. Predicting Adsorption Affinities of Small Molecules on Carbon Nanotubes Using Molecular Dynamics Simulation. ACS Nano 2015, 9, 11761-11774.

(24) Singam, E. R. A.; Zhang, Y.; Magnin, G.; Miranda-Carvajal, I.; Coates, L.; Thakkar, R.; Poblete, H.; Comer, J. Thermodynamics of Adsorption on Graphenic Surfaces from Aqueous Solution. J. Chem. Theory Comput. 2019, 15, 1302-1316.

(25) Sun, Z.; Zhang, Y.; Yu, H.; Yan, C.; Liu, Y.; Hong, S.; Tao, H.; Robertson, A. W.; Wang, Z.; Pádua, A. A. H. New Solvent-Stabilized Few-Layer Black Phosphorus for Antibacterial Applications. Nanoscale 2018, 10, 12543-12553.

(26) Wang, J.; Wolf, R. M.; Caldwell, J. W.; Kollman, P. A.; Case, D. A. Development and Testing of a General Amber Force Field. J. Comput. Chem. 2004, 25, 11571175 .

(27) Becke, A. Density-Functional ExchangeEnergy Approximation with Correct Asymptotic Behavior. Phys. Rev. A 1988, 38, 3098-3100.

(28) Becke, A. D. Density?Functional Thermochemistry. III. The Role of Exact Exchange. J. Chem. Phys. 1993, 98, 56485652 .

(29) Cioslowski, J. General and Unique Partitioning of Molecular Electronic Properties into Atomic Contributions. Phys. Rev. Lett. 1989, 62, 1469.

(30) Cioslowski, J. A New Population Analysis Based on Atomic Polar Tensors. J. Am. Chem. Soc.' 1989, 111, 8333-8336.

(31) Stephens, P. J.; Jalkanen, K. J.; Kawiecki, R. W. Theory of Vibrational Rotational Strengths: Comparison of a Priori Theory and Approximate Models. J. Am. Chem. Soc. 1990, 112, 6518-6529.
(32) Frisch, M. J.; Trucks, G. W.; Schlegel, H. B.; Scuseria, G. E; Robb, M. A.; Cheeseman, J. R.; Scalmani, G.; Barone, V.; Petersson, G. A.; Nakatsuji, H.; Li, X.; Caricato, M.; Marenich, A. V.; Bloino, J.; Janesko, B. G.; Gomperts, R.; Mennucci, B.; Hratchian, H. P.; Ortiz, J. V.; Izmaylov, A. F.; Sonnenberg, J. L.; Williams-Young, D.; Ding, F.; Lipparini, F.; Egidi, F.; Goings, J.; Peng, B.; Petrone, A.; Henderson, T.; Ranasinghe, D.; Zakrzewski, V. G.; Gao, J.; Rega, N.; Zheng, G.; Liang, W.; Hada, M.; Ehara, M.; Toyota, K.; Fukuda, R.; Hasegawa, J.; Ishida, M.; Nakajima, T.; Honda, Y.; Kitao, O.; Nakai, H.; Vreven, T.; Throssell, K.; Jr., J. A. M.; Peralta, J. E.; Ogliaro, F.; Bearpark, M. J.; Heyd, J. J.; Brothers, E. N.; Kudin, K. N.; Staroverov, V. N.; Keith, T. A.; Kobayashi, R.; Normand, J.; Raghavachari, K.; Rendell, A. P.; Burant, J. C.; Iyengar, S. S.; Tomasi, J.; Cossi, M.; Millam, J. M.; Klene, M.; Adamo, C.; Cammi, R.; Ochterski, J. W.; Martin, R. L.; Morokuma, K.; Farkas, O.; Foresman, J. B.; Fox, D. J. Gaussian 16, Revision B.01. Gaussian, Inc., Wallingford CT 2016,

(33) Abascal, J. L. F.; Vega, C. A General Purpose Model for the Condensed Phases of Water: TIP4P/2005. J. Chem. Phys. 2005, 123, 234505.

(34) Plimpton, S. Fast Parallel Algorithms for Short-Range Molecular Dynamics. J. Comput. Phys. 1995, 117, 1-19.

(35) Ryckaert, J.-P.; Ciccotti, G.; Berendsen, H. J. C. Numerical Integration of the Cartesian Equations of Motion of a System With Constraints: Molecular Dynamics of n-Alkanes. J. Comput. Phys. 1977, 23, 327-341.

(36) Nosé, S.; Klein, M. Constant Pressure Molecular Dynamics for Molecular Systems. Mol. Phys. 1983, 50, 1055-1076. 
(37) Nosé, S. A Molecular Dynamics Method for Simulations In the Canonical Ensemble. Mol. Phys. 1984, 52, 255-268.

(38) Hockney, R. W.; Eastwood, J. W. Computer Simulation Using Particles; CRC Press, 1988.

(39) Pollock, E. L.; Glosli, J. Comments on P3M, FMM, and the Ewald Method for Large Periodic Coulombic Systems. Comput. Phys. Commun. 1996, 95, 93-110.

(40) Bunn, C. The Crystal Structure of LongChain Normal Paraffin Hydrocarbons. The Shape of the CH2 Group. Trans. Faraday Soc. 1939, 35, 482-491.

(41) Fu, H.; Shao, X.; Chipot, C.; Cai, W. Extended Adaptive Biasing Force Algorithm. An On-the-Fly Implementation for Accurate Free-Energy Calculations. J. Chem. Theory Comput. 2016, 12, 3506-3513.

(42) Lesage, A.; Lelièvre, T.; Stoltz, G.; Hénin, J. Smoothed Biasing Forces Yield Unbiased Free Energies with the Extended-System Adaptive Biasing Force Method. J. Phys. Chem. B 2017, 121, 3676-3685.

(43) Fu, H.; Zhang, H.; Chen, H.; Shao, X.; Chipot, C.; Cai, W. Zooming across the Free-Energy Landscape: Shaving Barriers, and Flooding Valleys. J. Phys. Chem. Lett. 2018, 9, 4738-4745.

(44) Darve, E.; Pohorille, A. Calculating Free Energies Using Average Force. J. Chem. Phys. 2001, 115, 9169-9183.

(45) Chipot, C., Pohorille, A., Eds. Free Energy Calculations: Theory and Applications in Chemistry and Biology; Springer Series in Chemical Physics 86; Springer: Berlin ; New York, 2007; OCLC: ocm79447449.

(46) Darve, E.; Rodríguez-Gómez, D.; Pohorille, A. Adaptive Biasing Force Method for Scalar and Cector Free Energy Calculations. J. Chem. Phys. 2008, 128, 144120 .
(47) Comer, J.; Gumbart, J. C.; Henin, J.; Lelievre, T.; Pohorille, A.; Chipot, C. The Adaptive Biasing Force Method: Everything You Always Wanted to Know but Were Afraid To Ask. J. Phys. Chem. B 2015, 119, 1129-1151.

(48) Filho, E. D.; Brito, E. L.; Nogueira, D. O.; Fonseca, J. L. Thermal Degradation and Drug Sorption in Hybrid Interpolyelectrolyte Particles. Colloids Surfaces A Physicochem. Eng. Asp. 2021, 610, 125894 .

(49) Pranami, G.; Lamm, M. H. Estimating Error in Diffusion Coefficients Derived from Molecular Dynamics Simulations. J. Chem. Theory Comput. 2015, 11, 45864592 . 
TOC Graphic

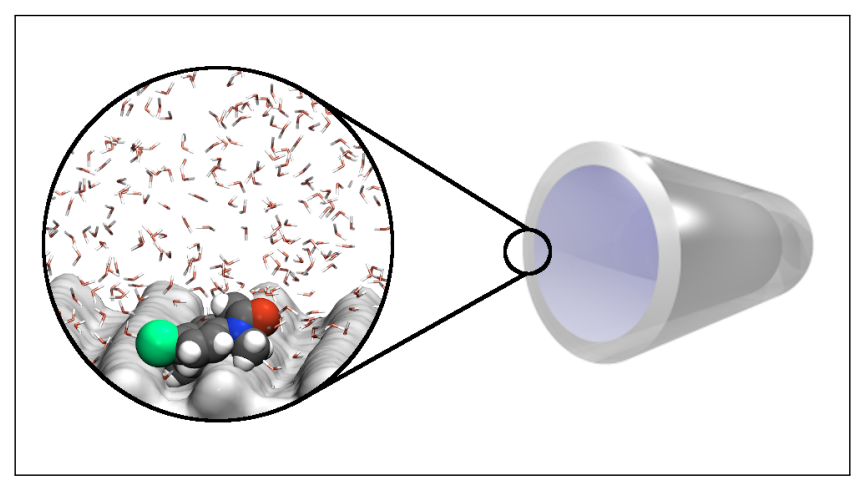

